Document downloaded from:

http://hdl.handle.net/10251/83531

This paper must be cited as:

Castelló-Palacios, S.; García Pardo, C.; Fornés Leal, A.; Cardona Marcet, N.; Vallés Lluch, A. (2016). Tailor-Made Tissue Phantoms Based on Acetonitrile Solutions for Microwave Applications up to $18 \mathrm{GHz}$. IEEE Transactions on Microwave Theory and Techniques. 64(11):3987-3994. doi:10.1109/TMTT.2016.2608890.

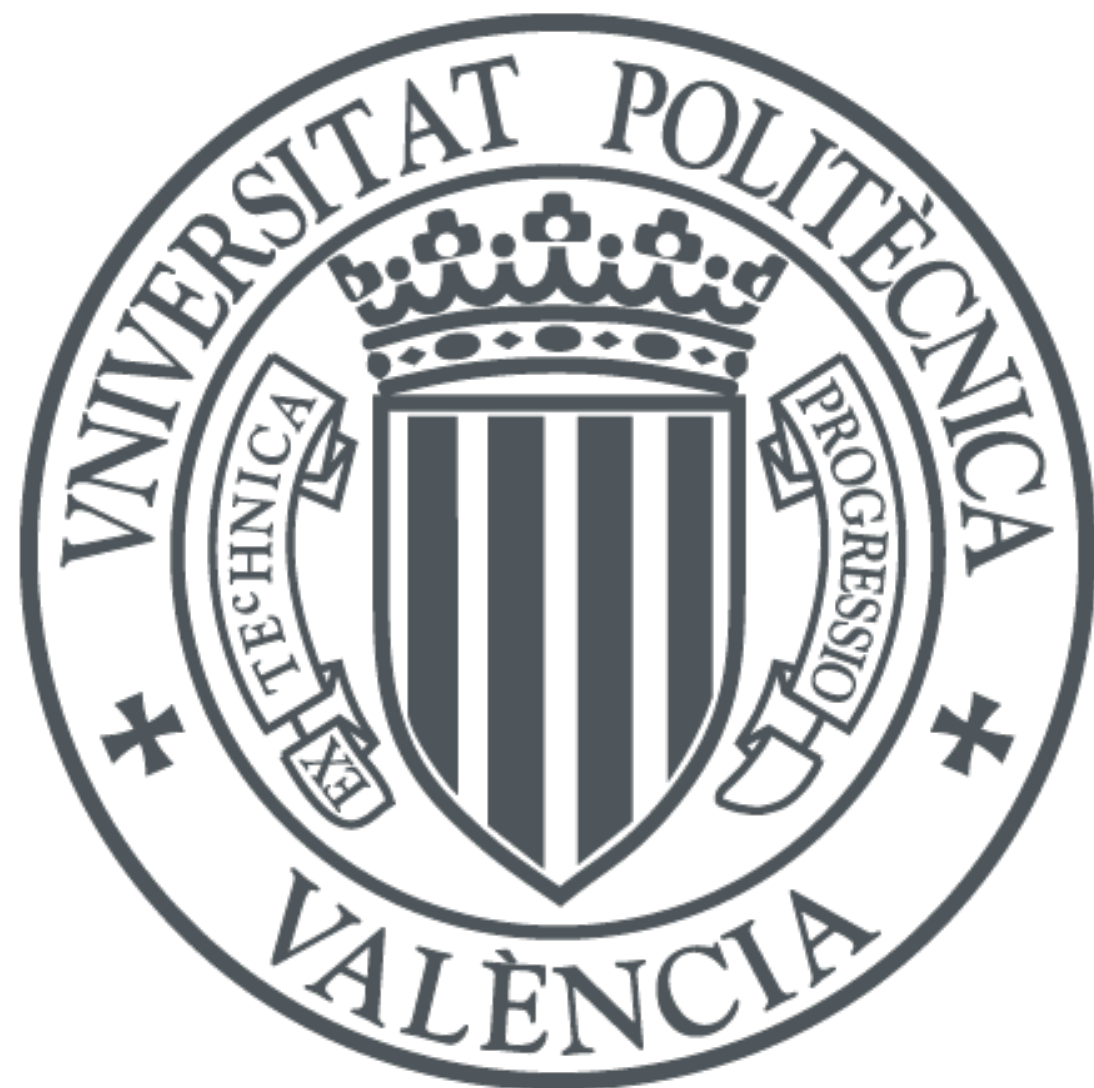

The final publication is available at

http://dx.doi.org/10.1109/TMTT.2016.2608890

Copyright Institute of Electrical and Electronics Engineers (IEEE)

Additional Information

(c) 2016 IEEE. Personal use of this material is permitted. Permission from IEEE must be obtained for all other users, including reprinting/ republishing this material for advertising or promotional purposes, creating new collective works for resale or redistribution to servers or lists, or reuse of any copyrighted components of this work in other works. 


\title{
Tailor-Made Tissue Phantoms Based on Acetonitrile Solutions for Microwave Applications up to $18 \mathrm{GHz}$
}

\author{
Sergio Castelló-Palacios, Concepcion Garcia-Pardo, Alejandro Fornes-Leal, Narcís Cardona, Senior \\ Member, IEEE, and Ana Vallés-Lluch
}

\begin{abstract}
Tissue-equivalent phantoms play a key role in the development of new wireless communications devices, which are tested on such phantoms prior to their commercialization. However, existing phantoms cover a small number of tissues and do not reproduce them accurately within wide frequency bands. This paper aims at enlarging the number of mimicked tissues as well as their working frequency band. Thus, a variety of potential compounds are scanned according to their relative permittivity from 0.5 to $18 \mathrm{GHz}$. Next, a combination of these compounds is characterized so the relation between their dielectric properties and composition is provided. Finally, taking advantage of the previous analysis, tailor-made phantoms are developed for different human tissues up to $18 \mathrm{GHz}$ and particularized for the main current Body Area Network (BAN) operating bands. The tailor-made phantoms presented here exhibit such a high accuracy that would allow researchers and manufacturers to test microwave devices at high frequencies for large bandwidths as well as the use of heterogeneous phantoms in the near future. The key of these phantoms lies in the incorporation of acetonitrile to aqueous solutions. Such compound has a suitable behavior to achieve the relative permittivity values of body tissues within the studied frequency band.
\end{abstract}

Index Terms-Acetonitrile, Body Area Network (BAN), Industrial, Scientific and Medical (ISM) band, microwave, tissueequivalent phantom, Ultra-Wideband (UWB).

\section{INTRODUCTION}

$M^{2}$ edical diagnoses based on electromagnetic techniques are widely present in different applications such as medical imaging or monitoring sensors. In all cases, electromagnetic waves travel through the human body to either analyze their variation due to tissue changes or transmit information between electronic devices. In this respect, the ongoing progress on getting smaller and low-power wireless devices allows them to be used in Body Area Networks (BANs) as integrated in-body sensors that continuously monitor health parameters [1], or ingestible capsules which go

This work was supported by the Ministerio de Economía y Competitividad, Spain (TEC2014-60258-C2-1-R), by the European FEDER funds.

S. Castelló-Palacios and A. Vallés-Lluch are with the Center for Biomaterials and Tissue Engineering, Universitat Politècnica de València, 46022 Valencia, Spain (e-mail: sercaspa@etsii.upv.es; avalles@ter.upv.es)

C. Garcia-Pardo, A. Fornes-Leal, and N. Cardona are with the iTEAM, Universitat Politècnica de València, 46022 Valencia, Spain (e-mail: cgpardo@iteam.upv.es; alforlea@iteam.upv.es; ncardona@iteam.upv.es). through internal cavities such as intestine [2]. Current technology is designed to use authorized frequency bands in order to connect sensors and receivers [3], but they are limited by very low rates of data transmission, high latency and low power efficiency [4]. Besides, most BANs work within the Industrial, Scientific and Medical (ISM) bands: 902 - 928 $\mathrm{MHz}, 2.4-2.4835 \mathrm{GHz}$ or $5.725-5.8255 \mathrm{GHz}$, which are narrowband and whose bit rate is not enough for future claimed applications. An alternative solution is UltraWideband (UWB), which is located within $3.1-10.6 \mathrm{GHz}$, a FCC specification with high data rates and low power consumption that takes advantage of bands wider than ISM [5].

Many antennas and in-body devices for BAN applications have been developed [6], [7], which are often tested in animals [8], [9] in order to improve their performance by adapting their design to the real transmitting medium, which is much more lossy than air. Tissue-equivalent materials, the so-called phantoms, are commonly used with the purpose of avoiding in vivo assays on humans or animals [10]-[12], inasmuch as the latter involve ethical and legal consequences as well as specimen dependence. These phantoms are supposed to exhibit the same dielectric properties, i.e., relative permittivity, as those of human tissues. There are both commercial and reported formulas for self-preparation phantoms [13], just as liquids [14], gel/semisolids [15], [16] or solids [17]. In the vast majority of cases, phantoms are made of ordinary ingredients such as water, sugar [18], salt, gelatinin-oil [19] or flour [20] with preservatives or combined with other compounds. Taking into account that relative permittivity is frequency-dependent, phantoms are usually provided for specific bands since it is not possible to imitate a tissue at every frequency. These are usually reported for narrow bands, e.g., ISM bands [13], with the aim of considering the relative permittivity constant because it does not suffer significant variations. Phantoms for wider bands have also been provided [21], but in most cases these phantoms do not imitate the entire UWB frequency band or own a quite weak approximation. Furthermore, there are not many mimicked tissues within wide frequency bands, which would be suitable to create heterogeneous phantoms that simulate a real environment for in-body applications. They are usually based on a general body area like head or torso. For instance, a well characterized head phantom is shown in [22] and [23], but only within $0.5-4 \mathrm{GHz}$. One critical example of 
the limitation of UWB phantoms is muscle, which is a key tissue for BANs and which has a fairly poor approximation [24]. In one of the frequently cited works [25], oil and water are combined in various ratios to match the dielectric trends of human tissues over a wide frequency range of $500 \mathrm{MHz}$ to 20 GHz. Nevertheless, this study is restricted to a few tissues with slight options to precise adjustment. Taking the same base ingredients, there have been attempts to improve this phantom formulation [26]. However, a solid material is offered, which is not a good option for wireless implanted sensors since they must often be placed inside the phantom. Besides, this phantom is not as accurate for a realistic equivalent of muscle above $5 \mathrm{GHz}$, because its conductivity is several units, in $\mathrm{S} / \mathrm{m}$, above that of the reference tissue. It is noteworthy to mention that most publications show the imaginary part of the relative permittivity as conductivity, where differences between values are lower than in loss factor and real deviation cannot be properly assessed.

The aim of this work is to develop a liquid synthetic model which can be tuned to meet the dielectric properties of the target tissue within $500 \mathrm{MHz}$ to $18 \mathrm{GHz}$, which comprise the future BAN bands like ISM or UWB. In addition, the model could be applied in other technologies, e.g., specific absorption rate (SAR) evaluation or microwave imaging, whose working frequencies are comprised within the same frequency range. To achieve this goal, it becomes imperative to analyze the behavior of different polar molecules because of their dipole orientation with the applied field, which is the predominant polarization type within the microwave band, so it determines the relative permittivity [27]. Solvents like water, acetone or acetonitrile own suitable properties, i.e., high dielectric constants and low polarization times [28], just like most human tissues. Ionic salts are also considered in this paper since they can provide extra conductivity to aqueous solutions.

This paper is organized as follows: in section II we describe the measurements and methodology carried out to prepare and characterize the liquid samples. Section III discusses the different chemical candidates for assembling phantoms. In section IV we obtain the spectrum of the achievable values for the relative permittivity, by combining the previously selected components, and then we provide some examples of targeted phantoms. Finally, section V summarizes the conclusions of our work.

\section{Measurement AND Methodology}

\section{A. Measurement system}

The measurement system used to characterize the relative permittivity is based on the open-ended coaxial probe method [29], [30]. It consists of a vector network analyzer (VNA, N9918A FieldFox Handheld Microwave Analyzer, frequency range: $30 \mathrm{kHz}-26.5 \mathrm{GHz}$ ), a $1 \mathrm{~m}$ long coaxial cable, a coaxial probe (Keysight $85070 \mathrm{E}$ slim form probe, working frequency: $500 \mathrm{MHz}-50 \mathrm{GHz}$ ), and a computer that managed VNA and processed the data. This system measures the reflection coefficient $\left(S_{11}\right)$ of the sample under test and translates it into the two parts of the complex relative permittivity (1):

$$
\varepsilon_{r}=\varepsilon_{r}^{\prime}-j \varepsilon_{r}^{\prime \prime}
$$

where $\varepsilon_{r}$ ' is the dielectric constant, and $\varepsilon_{r}$ " is the loss factor, both non-dimensional. This conversion is performed following the procedure described in [31], through self-created software.

The assembly was complemented with a hooked plier on a bracket, which held the slim probe, and a sample elevator, both with the aim of fixing the setup due to its high sensitivity. The full equipment setup is shown in Fig. 1.

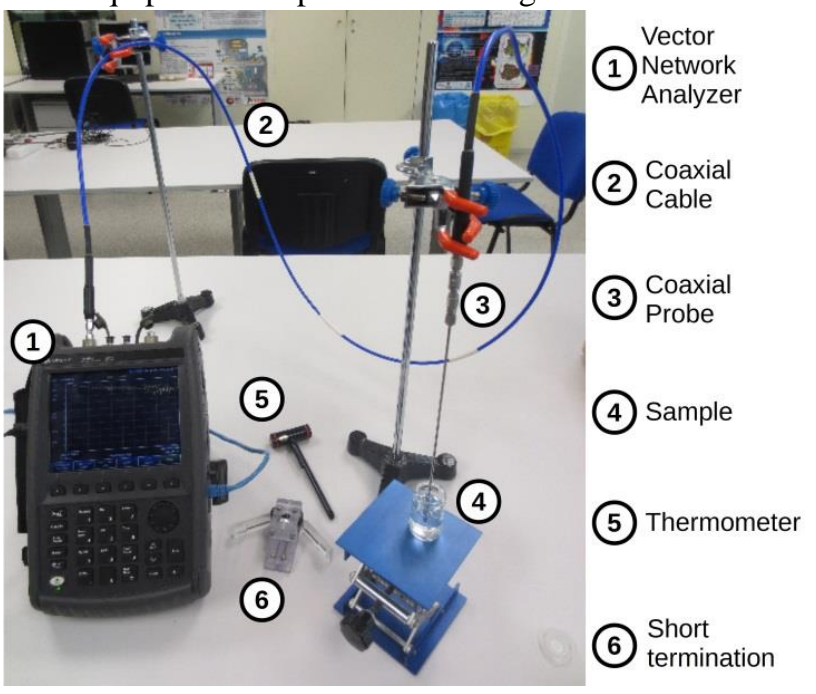

Fig. 1. Setup of the open-ended coaxial probe ready for measurements.

The measurement accuracy was established by comparing the results of a $0.1 \mathrm{M} \mathrm{NaCl}$ solution with those provided in [32], as suggested in [33]. The results show an uncertainty of $1.24 \%$ for the dielectric constant and $2.66 \%$ for the loss factor, in the frequency range from $500 \mathrm{MHz}$ to $10 \mathrm{GHz}$. Otherwise, from $10 \mathrm{GHz}$ to $18 \mathrm{GHz}$, there is an uncertainty of $1.31 \%$ for the dielectric constant and $3.34 \%$ for the loss factor. The main uncertainty terms are calibration and the effect of temperature.

\section{B. Methodology}

Regarding the liquid samples, they were prepared and measured inside glass vials as follows. Firstly, ionic solids were added, where appropriate, with a stainless steel lab spoon in the required quantities. Then, a Pasteur pipette was required to pour the polar liquids into the vials and fill them up to $20 \mathrm{~g}$ with deionized water. Next, the vials were closed and stirred for 15 minutes to ensure complete dissolution and mixture. Three replicates of each composition were prepared to minimize experimental errors.

The measurement system was configured as follows: the scanning range was comprised between $500 \mathrm{MHz}$ and 18 $\mathrm{GHz}$, recording 1601 points, so the frequency resolution was $10.9375 \mathrm{MHz}$. The IF bandwidth was set to $3 \mathrm{kHz}$ whereas the defined output power was $-3 \mathrm{dBm}$. A calibration process with well-known dielectric standards had to be set up with the purpose of removing the effect of fixture, cables and the coaxial probe so that the $S_{11}$ measurement and conversion could be performed properly. The calibration was carried out with air, deionized water, methanol (99.8\%, Sigma-Aldrich), and a short termination. Since VNA has drift errors due to temperature variation, the calibration process must be reset regularly; hence, it was performed every five scans. 
After calibration, it was important to avoid cable movements by using the sample elevator. Taking into account that the relative permittivity is temperature dependent, the samples were carefully measured at 24 degrees Celsius. Samples were characterized by immersing the probe inside the liquid halfway, removing air bubbles and ensuring an appropriate distance to the vial surface. After every measurement, the coaxial probe was cleaned with deionized water and smoothly dried with lab paper.

\section{MATERIALS SELECTION}

The procedure for achieving tissue phantoms was as follows: in the first step, aqueous solutions of polar liquids and ionic salts were analyzed with the aim of choosing those suitable to attain the dielectric response of human tissue according to literature [34]-[36]. Next, samples with a wide range of concentrations were prepared to disclose the achievable values of relative permittivity for designing phantoms. Finally, taking advantage of these results, ISM and UWB phantoms were synthesized according to the relationship between composition and relative permittivity.

Inasmuch as polar molecules are essential within the microwave band, it is mandatory to check them for any microwave application. Since the main compound of most tissues is water, it will work as the baseline for phantoms and be combined with other polar liquids. The review of polar compounds is performed with common chemicals. However, others such as N,N-dimethylformamide, nitromethane, N,Ndimethylacetamide or pyridine might be of interest. They are not studied here owing to their toxicity or unavailability. Fig. 2 shows water and aqueous solutions at $50 \%$ wt of different polar compounds in contrast to muscle, which is an extensible example of the behavior of tissues with high water content.

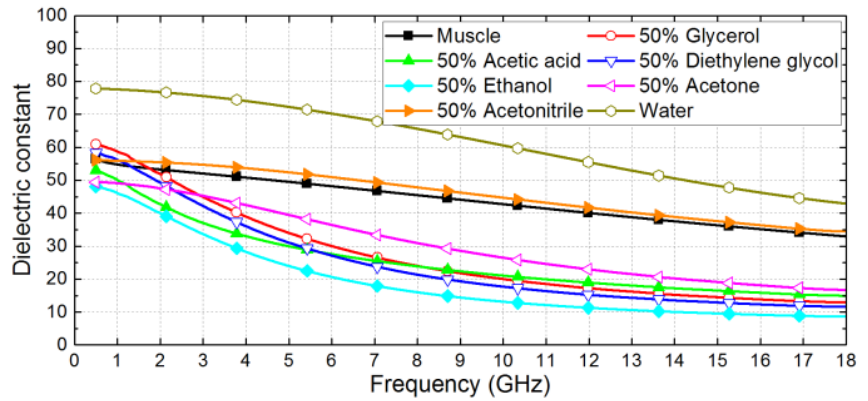

a)

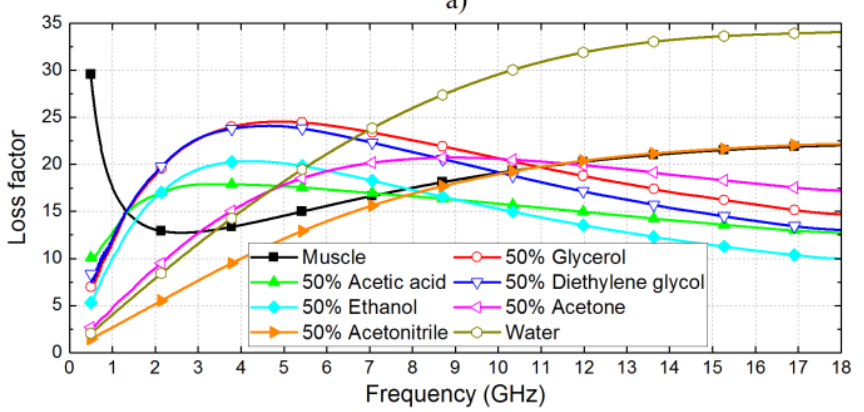

b)

Fig. 2. Relative permittivity of water, $50 \%$ wt aqueous mixtures of different polar liquids and that of muscle tissue [35]: a) Dielectric constant b) Loss factor.
All mixtures provoke a decrease from the water dielectric constant towards the range of tissue values around $500 \mathrm{MHz}$. The problem is that most of them show a high dielectric drop with frequency due to the relaxation frequency, from which molecules cannot orientate as fast as the electromagnetic field changes. Muscle, as well as many tissues, shows a soft decrease of the dielectric constant along frequency within the microwave band. However, the essential problem is not the dielectric constant but the loss factor, which exhibits a considerable growth around $4 \mathrm{GHz}$ when the relaxation frequency emerges and overtakes the muscle values; then, the loss factor falls again and deviates from tissue's behavior.

On the other hand, from these results, it can be stated that acetonitrile is the best candidate for phantoms since it has a strong similarity to muscle's behavior. It shows a very stable dielectric constant with frequency because its relaxation frequency, $45.734 \mathrm{GHz}$, is much larger than others, e.g., that of ethanol, $976.41 \mathrm{MHz}$ [28].

Regarding the loss factor, it should be increased between 0.5 and $9 \mathrm{GHz}$ to reach muscle values. For this purpose, salt has to be added as long as conductivity is defined by ionic polarization at frequencies below $10 \mathrm{GHz}$. Sodium chloride, a cheap and accessible salt typically proposed [26], [37], is compared in the form of an $2.5 \%$ wt aqueous solution against other salt solutions and pure water in Fig. 3, in order to select the best option.

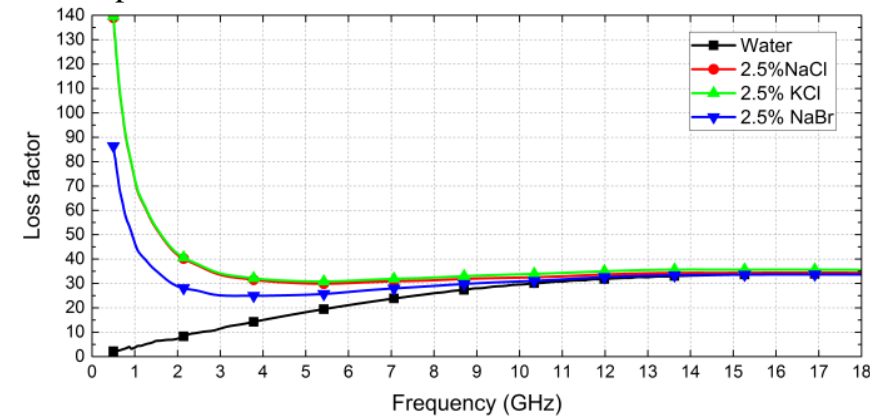

Fig. 3. Loss factor of $2.5 \%$ wt aqueous solutions of $\mathrm{NaCl}, \mathrm{KCl}$ and $\mathrm{NaBr}$ compared to deionized water.

As can be seen, choosing a salt is not critical inasmuch as all of them provide a huge increment on the loss factor below $10 \mathrm{GHz}$, higher than that pursued. The corresponding loss factor of potassium chloride and sodium chloride are almost the same and they overlap in the graph. Consequently, sodium chloride will be used hereafter in order to adjust the conductivity because it raises heavily the loss factor for a minimum quantity and it is the most easily available.

\section{PHANTOMS DESIGN}

\section{A. Composition scanning}

Four series of aqueous solutions with $30,40,50$, and $60 \%$ wt acetonitrile were prepared, since it is the proper candidate for preparing phantoms and therefore has to be studied in depth. Each series was replicated with varying $\mathrm{NaCl}$ concentrations from 0 to $5 \% \mathrm{wt}$ or until saturation point. The first series is shown in Fig. 4, corresponding to $30 \%$ wt of acetonitrile. 


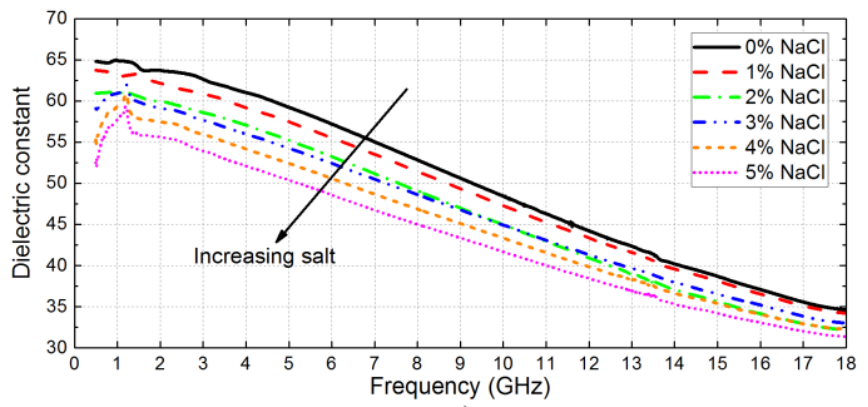

a)

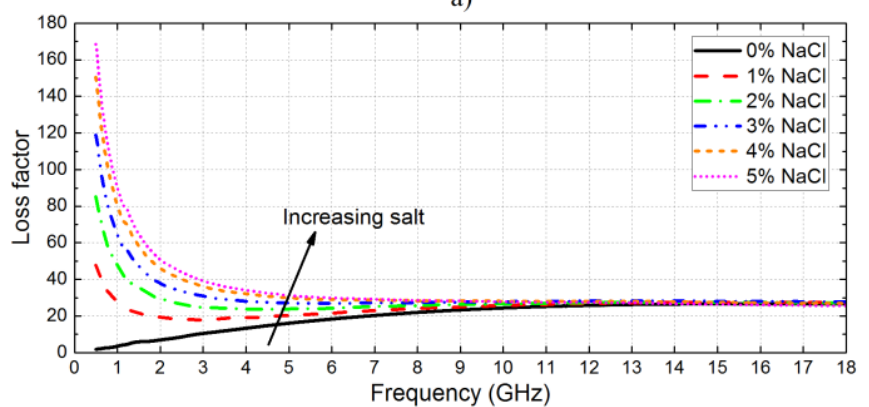

b)

Fig. 4. Relative permittivity of $30 \%$ wt acetonitrile aqueous solutions with different concentrations of $\mathrm{NaCl}$ : a) Dielectric constant b) Loss factor.

The presence of sodium chloride decreases the dielectric constant proportionally at any frequency. This fact can be explained by the smallness of its dissociated ions, which do not affect the orientation of polar molecules, so the relaxation frequency does not change. Indeed, the fall of the constant is due to the reduction of polar molecules per volume unit. In relation to the loss factor, the presence of ions raises noticeably its value below $10 \mathrm{GHz}$ as expected because of electrical conductivity.

Next series is presented in Fig. 5, where $40 \%$ wt acetonitrile in water is combined with $\mathrm{NaCl}$.
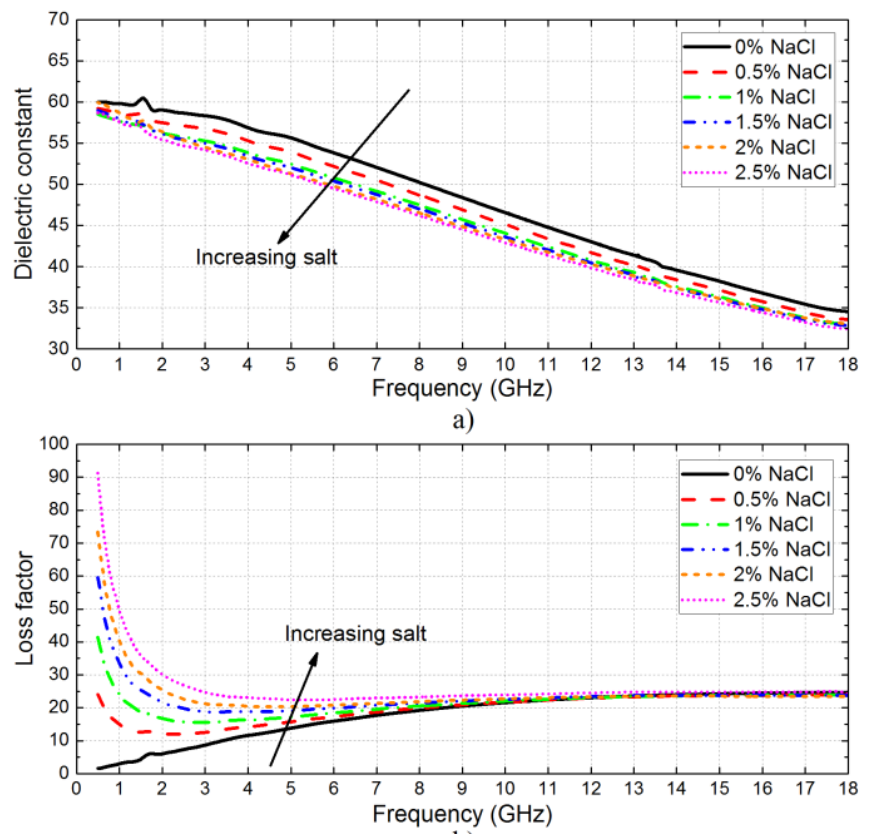

b)

Fig. 5. Relative permittivity of $40 \%$ wt acetonitrile aqueous solutions with different weight concentrations of $\mathrm{NaCl}$ : a) Dielectric constant b) Loss factor.
The values of the dielectric constant have decreased a few units with respect to the previous series. As in the previous case, the addition of salt decreases the dielectric constant without changing its behavior within the frequency band and increases the loss factor below $6 \mathrm{GHz}$.

The role of acetonitrile is better appreciated in Fig. 6, which displays the relative permittivity of $50 \%$ wt acetonitrile solutions. In this case, we can see the influence of acetonitrile on the dielectric constant, which gets more stable values (note the $y$-axis) with frequency due to its higher relaxation frequency in comparison with water. This is a highly significant finding for wideband phantoms since human tissues behave in that way (Fig. 2).
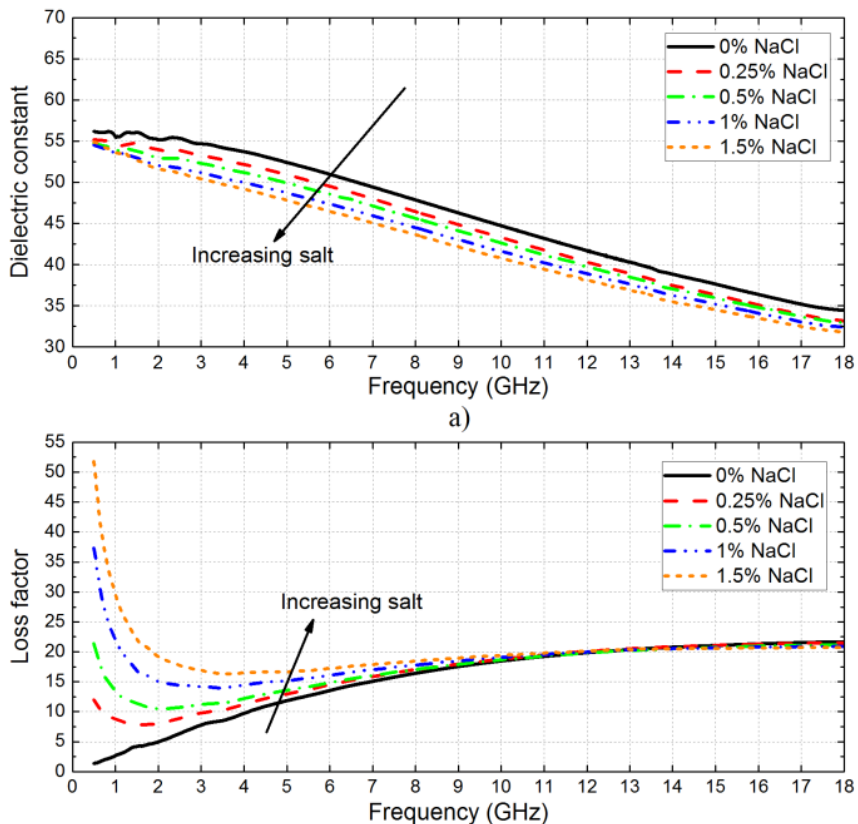

b)

Fig. 6. Relative permittivity of $50 \%$ wt acetonitrile aqueous solutions with different weight concentrations of $\mathrm{NaCl}$ : a) Dielectric constant b) Loss factor.

In this case, dissolving $2 \%$ wt $\mathrm{NaCl}$ was not possible since it exceeds the saturation point of the solution, so it was discarded. As it can also be observed from Fig. 6, the effect of the salt addition is similar regardless of the acetonitrile concentration.

The most concentrated aqueous solutions, i.e., those with $60 \%$ wt acetonitrile, are given in Fig. 7. Once again, the curves of the dielectric constant show a gentler slope with frequency compared to the previous cases with less acetonitrile in the formulation. The decrease of the dielectric constant with the rise of concentration can be noted in the $y-$ axis, which is kept in these figures to compare better. It should be remarked that the loss factor decreases with the increment of acetonitrile. This issue can be solved at frequencies below $10 \mathrm{GHz}$ by adding sodium chloride until saturation point. However, above $10 \mathrm{GHz}$ the effect of salt disappears and the loss factor cannot be modified without changing the concentration of polar liquids. It should be noticed that the fluctuation in the loss factor caused by the addition of salt is significant at frequencies below $2 \mathrm{GHz}$ due to the exponential trend of the curves. Consequently, it will be challenging to adjust the loss factor in phantoms for these frequencies. 


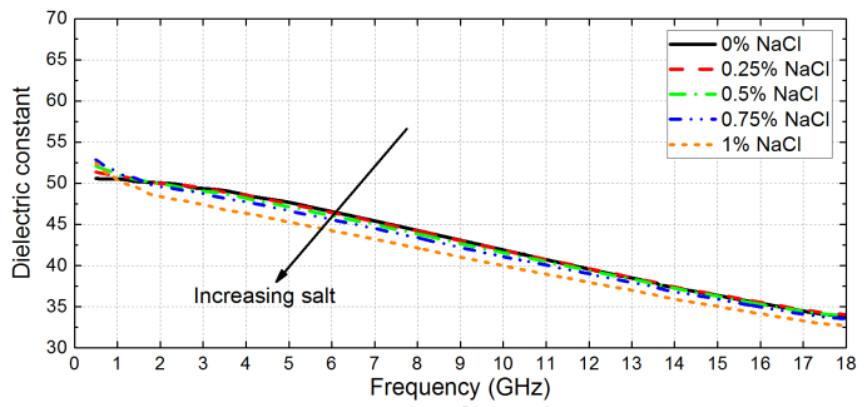

a)

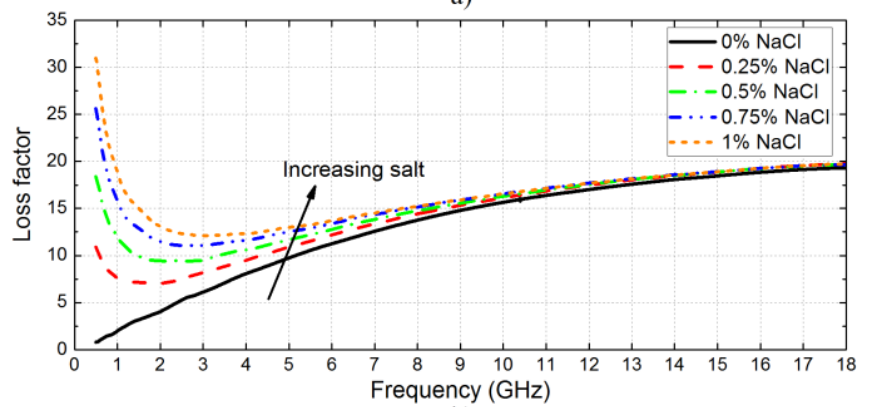

b)

Fig. 7. Relative permittivity of $60 \%$ wt acetonitrile aqueous solutions with different weight concentrations of $\mathrm{NaCl}$ : a) Dielectric constant b) Loss factor.

In summary, acetonitrile provokes a rise on the relaxation frequency of water that has an effect on both dielectric constant and loss factor. Regarding the dielectric constant, there is a decrease of its value because of the lower polarization capability of acetonitrile compared to water molecules. Besides, its stable value with frequency is improved, what is highly relevant for mimicking the tissues trend. This increase in relaxation frequency is also determinant in the loss factor, which is reduced at any frequency since the maximum of the loss is shifted to higher frequencies. From these systematized results it is possible to approach the required composition for a tissue phantom by analyzing and interpolating the response of the relative permittivity on the presented graphs for the required frequency band.

\section{B. Tissue Phantoms Tailoring}

Taking advantage of the preceding results, empirical attempts to imitate different tissues in the UWB frequency band were performed. The targeted tissues were mainly those of the torso, which may be relevant in BAN applications. After referring to the bibliographic data of real tissues [38], the targeted values were adjusted in the previous graphs at 4 and $8 \mathrm{GHz}$, and then adjusted with trial and error method. The choice of these frequencies is due to the fact that they are near the beginning and end of the UWB frequency band. Any other pair of frequencies within this band could be applicable, since the relative permittivity of human tissues holds a nearly linear behavior in this band [34]. Hence, we can assume that phantoms will be proper if they imitate the tissues at those frequencies. Despite phantoms were measured at 24 degrees Celsius, they were compared with the relative permittivity of the tissues at body temperature. Thus, phantoms at room temperature imitate the tissues within their real environment.

In Fig. 8, the relative permittivity for the phantoms designed for muscle $(54.98 \%$ Acetonitrile, $1.07 \% \mathrm{NaCl})$, heart $(49.94 \%$ Acetonitrile, $1.58 \% \mathrm{NaCl}$ ) and pancreas $(44.49 \%$ Acetonitrile,
$1.09 \% \mathrm{NaCl})$ are presented in contrast to those of mimicked human tissues [38] and a previously proposed phantom, 1M sucrose in water [24].

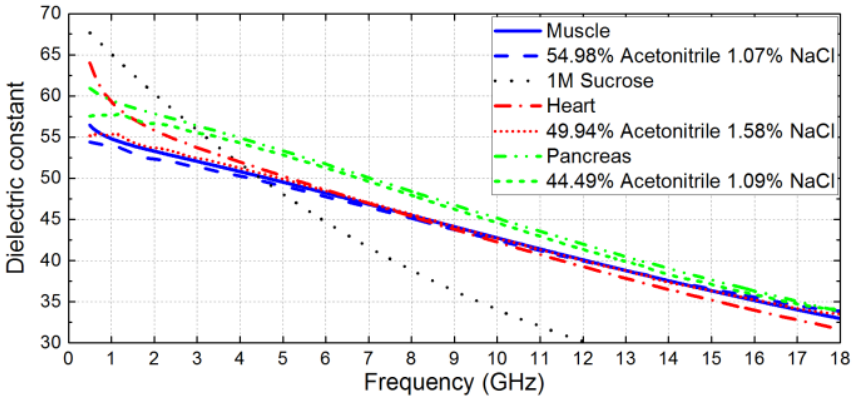

a)

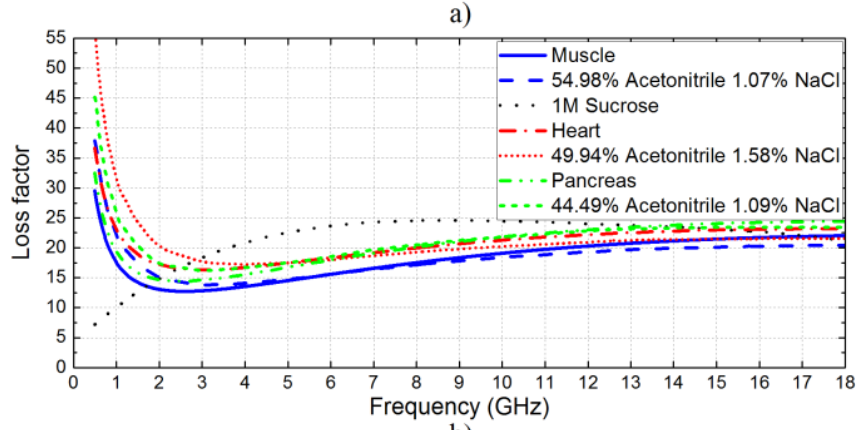

b)

Fig. 8. Relative permittivity of the muscle, heart and pancreas phantoms compared to $1 \mathrm{M}$ sucrose solution and the corresponding human tissues [35]: a) Dielectric constant b) Loss factor.

As can be seen, the above presented scans lead to liquid phantoms which mimic thoroughly tissues with high water content such as muscle, heart, pancreas, etc. In all cases, the reached accuracy is much higher than that already reported in bibliography for UWB, e.g., 1M sucrose, inasmuch as not only the curve trends are imitated but value deviations are negligible at both, real and imaginary parts within this band. There is a deviation for the relative permittivity at frequencies below $3 \mathrm{GHz}$ because UWB has been the focused band, regardless of a lower approximation within other bands, due to its relevance in BANs. The significant finding is the coincident trend between phantoms and tissues, which gives the possibility for an accurate reproduction within the entire frequency range that can be adjusted according to the demanded band.

Some tissues such as liver or cartilage have values of relative permittivity which are out of those that can be reached by using only acetonitrile. Besides, other tissues like colon have a quite marked fall of the dielectric constant with frequency so the phantom deviates from it when frequency rises. The solution for these tissues is the incorporation of another polar component in the solution apart from acetonitrile: ethanol. The latter enhances the possibility of preparing phantoms with less content of water since it has lower values of dielectric constant and relaxation frequency. In Fig. 9, the colon (48.5\% Acetonitrile, 2.5\% Ethanol, $1.165 \% \mathrm{NaCl}$ ), liver (51\% Acetonitrile, 17\% Ethanol, 0.95\% $\mathrm{NaCl})$ and cartilage phantoms (41\% Acetonitrile, 30\% Ethanol, $1 \% \mathrm{NaCl}$ ), which require increasing amounts of ethanol, can be compared with their homologous human tissues, taken from [38]. 

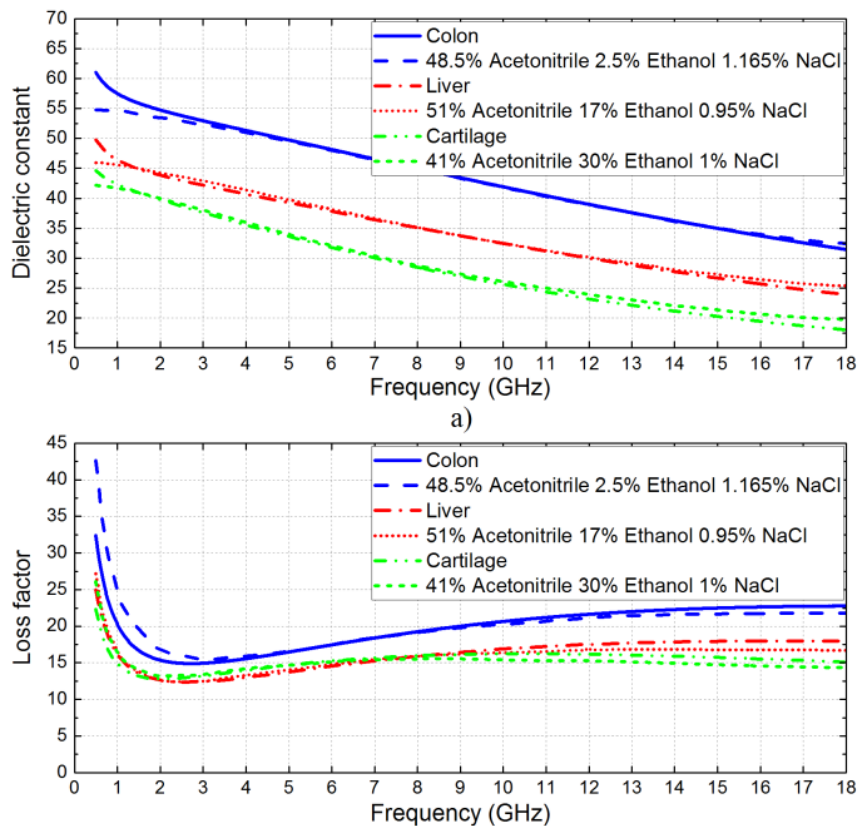

b)

Fig. 9. Relative permittivity of the colon, liver and cartilage phantoms compared to the human tissue [38]: a) Dielectric constant b) Loss factor.

It is important to keep in mind that the addition of ethanol decreases the solvation of sodium chloride, i.e., a lesser amount of salt can be added until its saturation point. Once it is exceeded, two phases split so the phantom is no longer homogeneous and has to be discarded. Hence, the loss factor may not be reached in such phantoms which require large ethanol contents for achieving low dielectric constants. Besides, an increment of ethanol reduces the relaxation frequency, i.e., there is a stronger fall of the dielectric constant with frequency. On the other hand, the maximum of the loss factor approaches the studied band (Fig. 2), moving away from the tissue's trend if the amount of ethanol is too much.

In summary, Table I collects the compositions of the presented phantoms, which are especially suitable between 3 and $18 \mathrm{GHz}$. The choice of this frequency range is justified by the fact that it comprises the entire UWB frequency band, the future wideband technology for BANs.

TABLE I

PHANTOM COMPOSITIONS IN THE 3-18 GHZ FREQUENCY BAND

\begin{tabular}{cc}
\hline \hline Tissue & Phantom composition \\
\hline Muscle & $54.98 \%$ Acetonitrile, $1.07 \% \mathrm{NaCl}$ \\
Heart & $49.94 \%$ Acetonitrile, $1.58 \% \mathrm{NaCl}$ \\
Pancreas & $44.49 \%$ Acetonitrile, $1.09 \% \mathrm{NaCl}$ \\
Colon & $48.5 \%$ Acetonitrile, $2.5 \%$ Ethanol, $1.165 \% \mathrm{NaCl}$ \\
Liver & $51 \%$ Acetonitrile, $17 \%$ Ethanol, $0.95 \% \mathrm{NaCl}$ \\
Cartilage & $41 \%$ Acetonitrile, $30 \%$ Ethanol, $1 \% \mathrm{NaCl}$ \\
\hline \hline
\end{tabular}

For the purpose of imitating properly some tissues within the 2.4-2.4835 GHz ISM band, different solutions from those shown above should be prepared. Table II lists the values obtained for ISM phantoms in contrast to the real tissue values, taken from [38]. Ethanol has been incorporated here as well to better mimic tissues such as colon and liver.
TABLE II

ISM (2.4-2.4835 GHz) PHANTOMS AT 24 DEGREES CELSIUS COMPARED WITH THEIR RESPECTIVE HUMAN TISSUES AT BODY TEMPERATURE [38]

\begin{tabular}{|c|c|c|c|c|c|c|c|}
\hline \multirow{2}{*}{ Tissue } & \multirow{2}{*}{$\begin{array}{l}\text { Phantom } \\
\text { composition }\end{array}$} & \multicolumn{3}{|c|}{ Dielectric constant } & \multicolumn{3}{|c|}{ Loss factor } \\
\hline & & Real & Phantom & Error & Real & Phantom & Error \\
\hline Heart & $\begin{array}{c}48.56 \% \\
\text { Acetonitrile, } \\
1.24 \% \mathrm{NaCl}\end{array}$ & 54.92 & 54.24 & $1.24 \%$ & 16.6 & 16.83 & $1.39 \%$ \\
\hline Muscle & $\begin{array}{c}55.05 \% \\
\text { Acetonitrile, } \\
0.86 \% \mathrm{NaCl}\end{array}$ & 52.79 & 51.94 & $1.61 \%$ & 12.77 & 12.78 & $0.08 \%$ \\
\hline Pancreas & $\begin{array}{c}44.48 \% \\
\text { Acetonitrile, } \\
0.84 \% \mathrm{NaCl}\end{array}$ & 57.27 & 56.54 & $1.27 \%$ & 14.44 & 14.39 & $0.35 \%$ \\
\hline Liver & $\begin{array}{c}17.5 \% \text { Ethanol, } \\
50 \% \\
\text { Acetonitrile, } \\
1 \% \mathrm{NaCl}\end{array}$ & 43.12 & 43.97 & $1.97 \%$ & 12.38 & 12.54 & $1.29 \%$ \\
\hline Colon & $\begin{array}{c}51.46 \% \\
\text { Acetonitrile, } \\
1.08 \% \mathrm{NaCl}\end{array}$ & 53.97 & 53.31 & $1.22 \%$ & 14.98 & 15.03 & $0.33 \%$ \\
\hline Cartilage & $\begin{array}{c}30 \% \text { Ethanol, } \\
45 \% \\
\text { Acetonitrile, } \\
1.25 \% \mathrm{NaCl}\end{array}$ & 38.88 & 38.39 & $1.26 \%$ & 12.86 & 12.95 & $0.70 \%$ \\
\hline
\end{tabular}

These phantoms at 24 degrees Celsius imitate properly the relative permittivity of tissues at body temperature. Anyway, their variation with temperature was checked between 20 and 40 degrees Celsius and it resulted in around \pm 0.08 units per degree for the dielectric constant, and \pm 0.11 units per degree for the loss factor. It is a deviation of $0.22 \%$ and $1.71 \%$ for both parts, respectively. The synthetic models are suitable for the assessment of many microwave technologies that transmit wireless waves throughout the human body [39].

These phantoms are homogeneous liquids with a quick and simple preparation. This fact provides them with a huge adaptability for different applications inasmuch as they can be placed in manifold containers. For instance, the phantom could be held within a 3D-printed plastic recipient with the shape of any tissue or embedded in a hydrogel. Thus, heterogeneous models may be prepared by placing the single phantoms in the required distribution once they are held in their corresponding containers. In addition, they were checked after three months stored within glass vials and no changes in their dielectric response were observed.

\section{CONCLUSIONS}

We have measured and processed data of the relative permittivity for many solutions with salts and polar molecules in order to choose the appropriate ones to be included in tissue phantoms. After the selection, these compounds have been systematically combined at increasing concentrations so that a relation between compositions and their dielectric properties has been provided. Then, several phantoms have been designed, bearing in mind the behavior of the tissue to be mimicked over frequency. Human tissues with different content of water were properly imitated in the frequency range between 3 and $18 \mathrm{GHz}$, assuming that the true dielectric properties of tissues are those reported in [38]. Since these phantoms are characterized at 24 degrees Celsius, any test with them should be carried out at this temperature.

This is a significant step for microwave applications, especially those that make use of wide bands difficult to be imitated to its full extent with one single phantom. Besides, 
this model is useful to get narrow band phantoms that are suitable for ISM and other technologies. The possibility of enlarging the phantom repertoire is noticeable, and it will be carried out in further works. Other issues to be addressed are the shelf life and compatibility of these phantoms with different containers. Anyway, their components are miscible, and so no phase separation or sediment appears over time.

\section{REFERENCES}

[1] B. Latré, B. Braem, I. Moerman, C. Blondia, and P. Demeester, "A survey on wireless body area networks," Wirel. Networks, vol. 17, no. 1, pp. 1-18, Jan. 2011.

[2] A. Moglia, A. Menciassi, M. O. Schurr, and P. Dario, "Wireless capsule endoscopy: From diagnostic devices to multipurpose robotic systems," Biomed. Microdevices, vol. 9, no. 2, pp. 235-243, Apr. 2007.

[3] S. Ullah, H. Higgins, B. Braem, B. Latre, C. Blondia, I. Moerman, S. Saleem, Z. Rahman, and K. S. Kwak, "A comprehensive survey of wireless body area networks on PHY, MAC, and network layers solutions," J. Med. Syst., vol. 36, no. 3, pp. 1065-1094, Jun. 2012.

[4] M. Chen, S. Gonzalez, A. Vasilakos, H. Cao, and V. C. M. Leung, "Body area networks: A survey," Mob. Networks Appl., vol. 16, no. 2, pp. 171-193, Apr. 2011.

[5] R. Aiello, A. Batra, and R. Sutton, Ultra Wideband Systems: Technologies and Applications, 1st ed. USA: Newnes, 2006.

[6] N. Chahat, M. Zhadobov, R. Sauleau, and K. Ito, "A compact UWB antenna for on-body applications," IEEE Trans. Antennas Propag., vol. 59, no. 4, pp. 1123-1131, Apr. 2011.

[7] E. Atashpanjeh and A. Pirhadi, "Design of Wideband Monopole Antenna Loaded with Small Spiral for Using in Wireless Capsule Endoscopy Systems," Prog. Electromagn. Res. C, vol. 59, pp. 71-78, Aug. 2015.

[8] D. Anzai, K. Katsu, R. Chavez-Santiago, Q. Wang, D. Plettemeier, J. Wang, and I. Balasingham, "Experimental evaluation of implant UWBIR transmission with living animal for body area networks," IEEE Trans. Microw. Theory Techn., vol. 62, no. 1, pp. 183-192, Jan. 2014

[9] C. Garcia-Pardo, R. Chavez-Santiago, N. Cardona, and I. Balasingham, "Experimental UWB frequency analysis for implant communications," in Proceedings of the Annual International Conference of the IEEE Engineering in Medicine and Biology Society (EMBC), Milan, Italy, Aug. 2015, pp. 5457-5460.

[10] R. Chavez-Santiago, C. Garcia-Pardo, A. Fornes-Leal, A. Valles-Lluch, G. Vermeeren, W. Joseph, I. Balasingham, and N. Cardona, "Experimental Path Loss Models for In-Body Communications within 2.36-2.5 GHz.," IEEE J. Biomed. Heal. informatics, vol. 19, no. 3, pp. 930-937, May 2015.

[11] H. Y. Lin, M. Takahashi, K. Saito, and K. Ito, "Performance of implantable folded dipole antenna for in-body wireless communication," IEEE Trans. Antennas Propag., vol. 61, no. 3, pp. 1363-1370, Mar. 2013.

[12] S. Jacobsen and P. R. Stauffer, "Multifrequency radiometric determination of temperature profiles in a lossy homogeneous phantom using a dual-mode antenna with integral water bolus," IEEE Trans. Microw. Theory Techn., vol. 50, no. 7, pp. 1737-1746, Jul. 2002.

[13] C.-K. Chou, G.-W. Chen, A. W. Guy, and K. H. Luk, "Formulas for preparing phantom muscle tissue at various radiofrequencies," Bioelectromagnetics, vol. 5, no. 4, pp. 435-441, 1984.

[14] G. A. Conway, W. G. Scanlon, C. Orlenius, and C. Walker, "In situ measurement of UHF wearable antenna radiation efficiency using a reverberation chamber," IEEE Antennas Wirel. Propag. Lett., vol. 7, pp. 271-274, Mar. 2008

[15] Y. Yuan, C. Wyatt, P. Maccarini, P. Stauffer, O. Craciunescu, J. Macfall, M. Dewhirst, and S. K. Das, "A heterogeneous human tissue mimicking phantom for RF heating and MRI thermal monitoring verification.," Phys. Med. Biol., vol. 57, no. 7, pp. 2021-2037, Mar. 2012.

[16] A. Dabbagh, B. J. J. Abdullah, C. Ramasindarum, and N. H. Abu Kasim, "Tissue-Mimicking Gel Phantoms for Thermal Therapy Studies," Ultrason. Imaging, vol. 36, no. 4, pp. 291-316, Mar. 2014.

[17] H. Tamura, Y. Ishikawa, T. Kobayashi, and T. Nojima, "A dry phantom material composed of ceramic and graphite powder," IEEE Trans. Electromagn. Compat., vol. 39, no. 2, pp. 132-137, May 1997.
[18] G. Hartsgrove, A. Kraszewski, and A. Surowiec, "Simulated biological materials for electromagnetic radiation absorption studies," Bioelectromagnetics, vol. 8, no. 1, pp. 29-36, 1987.

[19] A. Mashal, F. Gao, and S. C. Hagness, "Heterogeneous Anthropomorphic Phantoms with Realistic Dielectric Properties for Microwave Breast Imaging Experiments," Microw. Opt. Technol. Lett., vol. 53, no. 8, pp. 1896-1902, Aug. 2011.

[20] S. M. Salvador and G. Vecchi, "Experimental tests of microwave breast cancer detection on phantoms," IEEE Trans. Antennas Propag., vol. 57, no. 6, pp. 1705-1712, Jun. 2009.

[21] C. Gabriel, "Tissue Equivalent Material for Hand Phantoms," Phys. Med. Biol., vol. 52, no. 14, pp. 4205-4210, Jun. 2007.

[22] B. J. Mohammed and A. M. Abbosh, "Realistic Head Phantom to Test Microwave Systems for Brain Imaging," Microw. Opt. Technol. Lett., vol. 56, no. 14, pp. 979-982, Apr. 2014.

[23] A. T. Mobashsher and A. M. Abbosh, "Three-dimensional human head phantom with realistic electrical properties and anatomy," IEEE Antennas Wirel. Propag. Lett., vol. 13, pp. 1401-1404, Jul. 2014.

[24] H. Yamamoto, J. Zhou, and T. Kobayashi, "Ultra wideband electromagnetic phantoms for antennas and propagation studies," IEICE Trans. Fundam. Electron. Commun. Comput. Sci., vol. E91-A, no. 11, pp. 3173-3182, Nov. 2008.

[25] M. Lazebnik, E. L. Madsen, G. R. Frank, and S. C. Hagness, "Tissuemimicking phantom materials for narrowband and ultrawideband microwave applications.," Phys. Med. Biol., vol. 50, no. 18, pp. 42454258, Aug. 2005.

[26] T. Yilmaz, R. Foster, and Y. Hao, "Broadband tissue mimicking phantoms and a patch resonator for evaluating noninvasive monitoring of blood glucose levels," IEEE Trans. Antennas Propag., vol. 62, no. 6, pp. 3064-3075, Jun. 2014.

[27] A. R. von Hippel, Dielectric Materials and Applications. Boston: Artech House, 1954

[28] J. Barthel and R. Buchner, "High frequency permittivity and its use in the investigation of solution properties," Pure Appl. Chem., vol. 63, no. 10, pp. 1473-1482, Jan. 1991.

[29] A. Nyshadham, C. L. Sibbald, and S. S. Stuchly, "Permittivity Measurements using Open-Ended Sensors and Reference Liquid Calibration - An Uncertainty Analysis," IEEE Trans. Microw. Theory Techn., vol. 40, no. 2, pp. 305-314, Feb. 1992.

[30] M. A. Stuchly, T. W. Athey, G. M. Samaras, and G. E. Taylor, "Measurement of Radio Frequency Permittivity of Biological Tissues with an Open-Ended Coaxial Line: Part I," IEEE Trans. Microw. Theory Techn., vol. 30, no. 1, pp. 87-92, Jan. 1982.

[31] T. P. Marsland and S. Evans, "Dielectric measurements with an openended coaxial probe," IEE Proc. H Microwaves, Antennas Propag., vol. 134, no. 4, p. 341, Aug. 1987

[32] R. Buchner, G. T. Hefter, and P. M. May, "Dielectric Relaxation of Aqueous NaCl Solutions," J. Phys. Chem. A, vol. 103, no. 1, pp. 1-9, Jan. 1999.

[33] C. Gabriel and A. Peyman, "Dielectric measurement: error analysis and assessment of uncertainty," Phys. Med. Biol., vol. 51, no. 23, pp. 60336046, Oct. 2006.

[34] S. Gabriel, R. W. Lau, and C. Gabriel, "The dielectric properties of biological tissues: II. Measurements in the frequency range $10 \mathrm{~Hz}$ to 20 GHz," Phys. Med. Biol., vol. 41, pp. 2251-2269, Nov. 1996.

[35] C. Gabriel, S. Gabriel, and E. Corthout, "The dielectric properties of biological tissues: I. Literature survey," Phys. Med. Biol., vol. 41, no. 11, pp. 2231-2249, Nov. 1996.

[36] C. Gabriel, "Compilation of the Dielectric Properties of Body Tissues at RF and Microwave Frequencies," Report N.AL/OE-TR- 1996-0037, Occupational and environmental health directorate, Radiofrequency Radiation Division, Brooks Air Force Base, Texas (USA), Jun. 1996.

[37] C. Beck, S. Nagele, J. Nagel, H. Guth, U. Gengenbach, and G. Bretthauer, "Low-cost head phantom for the evaluation and optimization of RF-links in ophthalmic implants," Biomed. Eng. (NY)., vol. 58, Sep. 2013.

[38] D. Andreuccetti, R. Fossi, and C. Petrucci, "An Internet resource for the calculation of the dielectric properties of body tissues in the frequency range $10 \mathrm{~Hz} \quad-100$ GHz." [Online]. Available: http://niremf.ifac.cnr.it/tissprop/. IFAC-CNR, Florence (Italy), 1997. Based on data published by C.Gabriel et al. in 1996.

[39] N. Cardona, S. Castelló-Palacios, A. Fornes-Leal, C. Garcia-Pardo, and A. Vallés-Lluch, "Modelo sintético de tejidos biológicos para la evaluación de la transmisión inalámbrica de ondas electromagnéticas," ES Patent 2575 731, Dec. 22, 2015. [in Spanish] 